Aber zugleich ist in die Comédie herö̈que eine List eingebaut, die die Komödie für ewig (oder beinahe ewig, um nicht zu übertreiben) heroisch werden lässt. Denn alle medialen Umsetzungen des Textes werden letztlich dem (im Text dargestellten) Dichter die Treue beweisen, indem sie dessen Exaggerationen befolgen: Sie werden seine Nase zeigen und damit seinen Übertreibungen - so sehr diese ihn auch geradezu physiognomisch entstellen mögen - auf den Leim gegangen sein. Cyrano hat nämlich seine Entstellung gleich selbst besorgt. Er ist der Autor seiner Nase: »Il exagère« - »Er übertreibt«. Die Poesie ist somit auf ironische Weise die Mutter (und der Dichter damit der Vater) aller aus den intermedialen Übertragungen entspringenden, missgeborenen weil grotesken - Körper. Die (intermediale) Rezeptionsgeschichte des Textes wird selber grotesk.

Rostands Cyrano de Bergerac ist daher nicht nur literarisch, sondern auch kulturwissenschaftlich interessant. Das Stück reflektiert, heute gelesen, den historischen Ort der Literatur; und es problematisiert überdies, wie die geschlechtlichen und devianten und womöglich sogar grotesken Körper nicht an sich gegeben sind, sondern nicht aufhören, der (immer schnelleren) Abfolge der Medien zu entspringen. Es ruft dazu auf, diese Medien zu bejahen; aber es ruft auch dazu auf, sie zu untersuchen - nämlich im Hinblick auf die Weise, wie sie Erbe des Tropus der Übertreibung und der alten Sinnfigur der evidentia sind.

Dietmar Schmidt

\title{
Oralität, Bewusstsein, Schreiben. Rose Cellis Comme l'eau (1930)
}

In impliziter Abgrenzung von Descartes' cogito wird in dem 1930 erschienenen Roman Comme l'eau von Rose Celli (1895-1982 ${ }^{1}$ ) die Oralität als ein konstituierendes Element von Seinsbewusstsein vorgestellt:

Avant de continuer ce livre, je me suis cherchée, et voici: entre mes deux mâchoires, je me suis trouvée. Là se tient, en éveil, ma conscience d'être: ni dans le cerveau, ni dans les membres, ni dans le ventre, ni dans la poitrine, mais dans la bouche. [...] Dans ma

1. Die Ermittlung der Lebensdaten verdankt sich der persönlichen Auskunft des Neffen der Autorin, Jean Brua. 
bouche, [...] [la vie] est forcée de me révéler son goût. ${ }^{2}$ - Bevor ich an diesem Buch weiter geschrieben habe, habe ich mich gesucht und da: zwischen meinen beiden Kiefern habe ich mich gefunden. Dort befindet sich, wach, mein Bewusstsein zu sein: weder im Gehirn, noch in den Gliedern, noch im Bauch, noch in der Brust, sondern im Mund. [...] In meinem Mund ist [...] [das Leben] gezwungen, mir seinen Geschmack zu offenbaren.

Im Mund findet das Erzähl-Ich das Zentrum seines Bewusstseins. Dieses Bild stellt die rationalistische Perspektive auf den Kopf und erzeugt durch diesen, an einen anderen Ort gerrückten (einen sverrückten`), Sinn von Bewusstsein zu Beginn einen latent komischen Effekt auf die Leser. Das Wasser, von dem im Titel die Rede ist, ist zunächst >das Wasser des Mundes`, der Speichel, nach Julia Kristeva das »Abjekt«3", bei Celli ist er jedoch keineswegs mit der Empfindung des Abscheus verbunden. Die eigenartige Zwischenstellung als »ni sujet ni objet«4, "weder Teil des Körpers, noch davon getrennt «5 nimmt der Speichel aber auch in Cellis Text ein. Vom Mund als dem Ort, an dem Innen und Außen sich berühren, geht die Diskussion des Verhältnisses von Ich und Welt, Subjekt und Objekt aus. Zudem ist der Mund der Ort, an dem »les paroles se forment avant d'être écrites « ${ }^{6}$ - »die Worte entstehen, bevor sie geschrieben werden«, wodurch die Materialität der Sprache herausgestellt wird. Die Frage, wie bzw. ob menschliches Bewusstsein in einem literarischen Text darstellbar ist und worüber es sich überhaupt konstituiert, bildet den Kern des handlungsarmen, sprachlich sehr lyrischen Romans. Ein Erzähl-Ich unternimmt darin den Versuch, das Bewusstsein einer Figur, Odile, zu erkunden. Bemerkenswert an der Erzählsituation ist, dass diese Figur aus dem erzählenden Ich heraustritt, daher das Ich selbst sein müsste, was jedoch verneint wird. ${ }^{7}$ Diskutiert wird somit auch die Frage der Identität des (schreibenden) Subjekts.

Der heute nur noch in der Bibliothèque Nationale zugängliche Text, über dessen Autorin wenig bekannt ist, ${ }^{8}$ ragt aufgrund seiner

2. Rose Celli: Comme l'eau, Paris: Edition du Tambourin 1930, S. 36 . Übersetzung hier und im Folgenden A.K.

3. Vgl. Inge Suchsland: Julia Kristeva, Hamburg: Junius 1992, S. 122-125.

4. Vgl. Julia Kristeva: Pouvoirs de l'horreur, Paris: Seuil 1980, S. 9.

5. Vgl. Doris Feldmann/Sabine Schülting: »Julia Kristeva«, in: Ansgar Nünning (Hg.): Lexikon Literatur- und Kulturtheorie, Stuttgart: Metzler 1998, S. 286f., hier S. 287.

6. R. Celli: Comme l'eau, S. 76.

7. »Mais je parle ici d'Odile, non de moi.« - »Ich spreche hier jedoch von Odile, nicht von mir.« R. Celli: Comme l'eau, S. 60.

8. Rose Celli (geb. Brua) wuchs in Algerien auf, kam 1914 nach Frankreich, um die Ausbildung an der Ecole de Sèvres zu absolvieren, entschied sich nach dem Ab- 
Auseinandersetzung mit dem Verhältnis von Wahrnehmung und Schreiben und seiner die Fiktion bloßlegenden Erzählweisen aus der eher traditionellen Romanproduktion der französischen 1930er Jahre heraus. ${ }^{9}$ Mit dem Bild der "phrase comme un soleil « - des "Satzes wie eine Sonne ${ }^{10}{ }^{0}$ entwirft Celli ein Konzept »radialen « Schreibens, das sie gleichzeitig metafiktional zu explizieren und praktisch umzusetzen versucht. Das entstehende Buch soll sich in seiner Form einem imaginären anderen Schreibheft der fiktiven Autorin annähern und "sans commencement ni fin, avec un point central et des rayons, et un mouvement circulaire ${ }^{11}$ - »ohne Anfang und Ende, mit einem zentralen Punkt und Strahlen, und einer kreisrunden Bewegung « sein. Diese sradiale`Struktur, die quer läuft zur konventionellen, linearen Satzstruktur, kann in einem Prosatext nicht umgesetzt werden, ohne diesen in Konkrete Poesie zu verwandeln, was nicht geschieht. Celli nähert sich dem Konzept dennoch an, indem, anstatt einer kausallogisch entwickelten Geschichte, die »histoire de la conscience d'Odile ${ }^{12}$ - »die Geschichte von Odiles Bewusstsein« in Teilstücken ohne Beachtung einer Chronologie erzählt oder 'gezeigt` wird. Da diese Textfragmente wiederholt assoziativ vom gleichen Punkt - der Metapher des Mundes ausgehen, wird in einem weiter gefassten Sinn der svon einem Punkt strahlende Satz`durchaus realisiert. Nicht zuletzt wird damit eine literarische Entsprechung für die Funktionsweise des menschlichen Bewusstseins, in dem von einem Eindruck mehrere Assoziationen ausgehen können, gefunden, der die lineare Satzstruktur gerade nicht entspricht.

Durch die metafiktionalen Kommentare des Erzähl-Ichs, das sich zugleich als fiktive Autorin des Romans präsentiert, macht der Text unablässig auf seine sprachliche Verfasstheit aufmerksam. Ähnlich der Technik der mise en abyme ${ }^{13}$, besteht Comme l'eau aus zwei

schluss aber gegen den Lehrberuf, um Schriftstellerin zu werden. Weitere Texte von R. C.: Le châle indien (1931), Isola (1933), Ombre (1936), A l'envers du tapis (1936). Danach lassen sich nur noch Märchenadaptionen und Übersetzungen aus dem Englischen (Herman Melville, John Steinbeck, Charles Dickens, Virginia Woolf u.a.) nachweisen.

9. Eliane Tonnet-Lacroix spricht von einem »retour du réalisme qui marque les années trente«, dies.: La littérature française de l'entre-deux-guerres, Paris: Nathan 1993, S. 170.

10. R. Celli: Comme l'eau, S. 14.

11. Ebd., S. 25.

12. Ebd., S. 191.

13. Die Technik der mise en abyme setzt Gide in seinem Roman Les faux monnayeurs (1925), in dem »sich [...] die Narration selbst thematisch« wird, um. Vgl. Wilfried Engler: Französische Literatur im 20. Jahrhundert, Tübingen/Basel: UTB 1994, hier S. 226. 
miteinander verflochtenen Erzählebenen: die Ebene der Szenen aus Odiles Erleben und die der Reflexion des Schreibens, auf der die fiktive Autorin alternative Möglichkeiten, den Roman zu gestalten, vorstellt und verwirft, sich dadurch von bestimmten Erzählweisen distanzierend, etwa der des traditionellen allwissenden Erzählers, der alle Fäden des Geschehens in der Hand hält und die imaginären Raum-Zeitgerüste der Fiktion als Abbild der Wirklichkeit präsentiert. Das Ich in Comme l'eau ist eine Erzählinstanz, die verunsichern möchte. Es antizipiert die Erwartungen des Lesepublikums und spielt mit ihnen. Nicht eine »aventure d'amour« - ein »Liebesabenteuer" werde erzählt, sondern »la plus étrange aventure, l'aventure d'être ${ }^{14}$ - »das eigenartigste Abenteuer überhaupt, das Abenteuer zu sein«. Der Begriff der aventure verweist ironisch auf den höfischen Roman des Mittelalters. Ist dort die aventure ausdrücklich mit einem Auszug in die Welt verbunden, geht es bei Celli jedoch um eine Reise ins Innere: "Je marche en moi-même. - »Ich gehe in mir selbst. ${ }^{15}$ Nicht das äußere Ereignis ist von Interesse, sondern die Weise, wie es sich im Bewusstsein manifestiert: "Aussi ne partirai-je pas, pour ce voyage, d'un événement, mais de ce goût de l'eau dans ma bouche et de ce creux entre les deux mâchoires où gît, tandis que je vous parle, toute la conscience de mon être. ${ }^{16}$ - »So werde ich diese Reise nicht von einem Ereignis beginnen, sondern vom Geschmack des Wassers in meinem Mund und von dieser Höhle zwischen meinen beiden Kiefern, wo, während ich zu Ihnen spreche, das ganze Bewusstsein meines Seins ruht."

Allerdings ist in diesem Bild vom körperlich konstituierten Bewusstsein auch der Verweis auf die Sprache enthalten. Nicht nur ist der Mund auch der Ort der Sprachartikulation, das Seins-Bewusstsein ist zudem präsent, während das Ich spricht. Einerseits wird der Körper zwar explizit als »instrument de conscience" - "Instrument des Bewusstseins ${ }^{17}$ bezeichnet und der Geschmack des eigenen Speichels als Zentrum des Seins-Bewusstseins entdeckt, von dem aus >das Buch strahlen soll. ${ }^{18}$ Andererseits wird die Vorstellung des rein körperlichen Bewusstseins zugleich unterlaufen. So ist zwar von Gerüchen und Geschmack die Rede, die zu der körperlichen Reaktion der Speichelproduktion führen, doch sind es ausdrücklich die Wörter, die die Reaktion auslösen: "Odeurs et saveurs. Voilà les mots qui ont fait monter à ma bouche le sel et l'eau de la découverte. « ${ }^{19}$ Die bewusstseinskonsti-

14. R. Celli: Comme l'eau, S. 31.

15. Ebd., S. 93.

16. Ebd., S. 33.

17. Ebd., S. 92.

18. Vgl. ebd., S. 31.

19. Ebd. 
tuierende Qualität von Sprache ist hier mitformuliert und es bleibt uneindeutig, welcher Funktion, die im Bild des Mundes gefasst ist, der Vorzug gegeben wird.

In einem Denken, das im Schädel seingeschlossen ist, kann sich für Odile kein `Bewusst-Sein einstellen, weil der Bezug zum Außen fehlt. Der Mund dagegen ist eine "porte lumineuse ${ }^{20}$ - »eine leuchtende Tür«. Die Kritik an der Dominanz der Ratio und damit an der selbstbezüglichen Setzung des Subjekts, wird in diesem Bild besonders deutlich. Der Mund ist der Ort, an dem Grenze und Verbindung zwischen dem Selbst und der äußeren Welt körperlich erfahrbar werden, "la frontière qui sépare Odile du monde « und "la conjonction aiguë du goûteur et de la chose goûtée « ${ }^{21}$ - »die enge Verbindung des Schmeckenden und des geschmeckten Dings «. Celli entwirft eine Vorstellung von Subjektivität, die sich nicht über den Ausschluss von, sondern den (körperlichen) Bezug zur Welt herstellt. Nicht in einer reinen Innerlichkeit (wie bei Descartes), sondern sdurch die Welt ‘ kommt Odile zu sich: »Odile, quand elle veut se trouver, s'arrache d'elle et se jette au plus loin d'elle. Puis elle marche vers son lointain. Elle voit que, d'elle à elle, il y a la profondeur du monde. ${ }^{22}$ - »Wenn Odile sich finden möchte, reißt sie sich von sich los und wirft sich so weit wie möglich von sich. Dann läuft sie ihrem Entfernten entgegen. Sie sieht, dass von ihr bis zu ihr die Tiefe der Welt liegt. « Diese Vorstellung ist allerdings erneut ambivalent, denn sie lässt sich ebenso lesen als die Unmöglichkeit, je ‘bei sich selbst` zu sein, weil zwischen elle und elle immer die Welt (die Sprache) liegt.

Was die Vorstellung der Subjektkonstitution betrifft, ist in Cellis Entwurf neben der Selbstversicherung über die sur Welt offenen` Oralität - ich schmecke und spreche, also bin ich - auch der persönliche Bezug zum anderen von Bedeutung. ${ }^{23}$ Odile und ihr Freund François verschmelzen gerade nicht in der Liebe, sondern werden durch den Blick des anderen zum sselbständigen subjekt. ${ }^{24}$ Den anderen in seiner Einzigartigkeit zu ssehen<, ist jedoch nur in kurzen Momenten von hoher Intensität möglich. Odile nimmt François' "présence réelle« in einem jener sexistentiellen Erlebnisaugenblicke` wahr, die als moments of

20. Ebd., S. 76.

21. Ebd., S. 222.

22. Ebd., S. 192.

23. In der französischen Philosophie gewann die Frage der Leiblichkeit und des Verhältnisses zum anderen erst mit der »Wende zum Konkreten« im Laufe der 1930er Jahre an Bedeutung, sodass sich Cellis Text diesbezüglich als avantgardistisch im Wortsinn präsentiert. Vgl. Friedrich Hogemann: »Maurice Merleau-Ponty«, in: Bernd Lutz (Hg.): Philosophen Lexikon, Stuttgart: Metzler 1995, S. 596-598, hier S. 596.

24. R. Celli: Comme l'eau, S. 109. 
being oder moments of vision im Schreiben von Virginia Woolf zentral sind. ${ }^{25}$ Auch die Lichtmetaphern, die bei Woolf häufig im Zusammenhang mit den moments of vision auftreten, ${ }^{26}$ lassen sich bei Celli finden:

Cependant, cette perception de la présence réelle de François lui est permise parfois, en éclairs. [...] Présence réelle, soudain perçue, je ne peux pas mieux dire: »Il est autreque-moi. [...] Il est aussi absolu, aussi divin que moi.« [...] Elle lui dit: Toi. [...] [D]ans ces instants lumineux, elle le voit dans sa conscience à lui, dans son pouvoir. ${ }^{27}$ - Doch ist ihr diese Wahrnehmung von François' wirklicher Gegenwart manchmal, in einem Aufblitzen, erlaubt. [...] Plötzlich wahrgenommene, wirkliche Gegenwart, ich kann es nicht besser sagen: »Er ist anders-als-ich. Er ist genauso absolut, genauso göttlich wie ich.» [...] Sie sagt zu ihm: Du. [...] In diesen lichten Momenten sieht sie ihn in seinem eigenen Bewusstsein, in seiner Macht.

Die Bedingung der Momenthaftigkeit, an die das 'Sehen des anderen in seiner Einzigartigkeit gebunden ist, wird im Text explizit benannt. Es sind im Wortsinn svisionäre Momente`, die aus der »demi-cécité« "Halbblindheit« oder gar dem »aveuglement total ${ }^{28}$ - der »völligen Blindheit«, die den alltäglichen Umgang der (modernen) Menschen miteinander bestimmen, herausragen. Die Priorität der sinnlichen Wahrnehmung beschränkt sich in Cellis Text demnach nicht auf den Geschmackssinn. Intensive Erlebnisaugenblicke sind häufig mit der Wahrnehmung von Lichtverhältnissen verbunden. In einer Szene ist die Wahrnehmung von Gerüchen und Licht mit Odiles Empfinden szu sich selbst zu kommen ' gekoppelt. Das Licht, das »den Raum bis zum Rand anfüllt « ${ }^{29}$ assoziiert die Qualität von Wasser. Wenn Odile in diesem Raum sin sich selbst eintaucht‘, dann, weil das Licht hier "comme l'eau « beschaffen ist und weil Odile selbst swie Wasser ist. $^{30}$ Darauf verweist nicht zuletzt ihr Name, in dem eau [o] klanglich enthalten ist. Es zeigt sich, dass dass Odiles Eigenschaft »comme l'eau « zu sein, ein Bild dafür ist, dass es dem Ich unmöglich ist, sich im Schreiben selbst zu erfassen. In dem Moment, in dem Odile sin die Sprache eintrittı, erhält sie eine Eigendynamik. Je länger sie sich `im Schreibheft aufhältı, desto mehr entgleitet sie dem schreibenden Ich: "A moi-même, Odile échappe. A mesure que le cahier s'emplit, les faibles liens qui attachaient à moi cette femme se dénouent." - »Mir selbst entgleitet Odile. In

25. Vgl. Willi Erzgräber: Virginia Woolf, Tübingen, Basel: UTB 1993, S. 24.

26. Vgl. ebd., S. 23.

27. R. Celli: Comme l'eau, S. $183 \mathrm{f}$.

28. Ebd., S. 185.

29. Ebd., S. 98.

30. Ebd., S. 221. 
dem Maße, in dem sich das Heft füllt, lösen sich die schwachen Fäden auf, die mich mit dieser Frau verbanden. ${ }^{31}$

Das negative Gegenkonzept zur »présence réelle« stellen in Cellis Text die choses - Dinge dar. Steht die présence für den Subjektcharakter, so ist das Ding bildlicher Ausdruck der `Objektwerdung، und Entfremdung vom Anderen und von sich selbst:

La tête sur l'épaule de François rassasié, Odile, silencieusement, pleure. Le compagnon de son plaisir est là. Elle peut le toucher, comme une chose. Elle effleure du doigt sa joue, vraiment comme une chose, et il lui en vient un malaise singulier. [...] Ce soir, Odile a perdu la grâce. Pourquoi se sent-elle si loin de cet homme? Où est sa joie? [...] Ce soir, elle est une chose, elle aussi, qu'on peut examiner. ${ }^{32}$ - Den Kopf an der Schulter des gesättigten François, weint Odile lautlos. Da ist der Begleiter ihrer Lust. Sie kann inn berühren, wie ein Ding. Sie streicht mit dem Finger über seine Wange, wirklich wie über ein Ding, und sie spürt ein eigenartiges Unbehagen. [...] Heute Abend hat Odile ihre Anmut verloren. Warum fühlt sie sich so fern von diesem Mann? Wo ist ihre Lust? [...] Heute Abend ist auch sie ein Ding, das man untersuchen kann.

So stellen schließlich die Dinge selbst für Odile ein »danger mortel «33 eine »tödliche Gefahr« dar, weil ihr Objektcharakter Odiles Sein zu infizieren droht. Wenn Odile die Dinge »freilässt«,34 d.h. sie von ihrer Objekthaftigkeit befreit, bannt sie damit auch die Gefahr des eigenen Selbstverlusts, weil im Entwurf dieser Utopie allen Wesen und Dingen Subjektcharakter zugestanden wird. Odile tritt Objekten wie lebendigen Wesen gegenüber, sie streichelt ein Möbelstück, das sie angestoßen hat und beweint einen zerbrochenen Gegenstand wie einen verstorbenen Menschen. ${ }^{35}$ Denn nähert man sich den Dingen auf behutsame Weise, erscheinen sie, "wenn man Glück hat, in einem anderen Licht und lösen sich auf «. ${ }^{36}$ Sie verlieren ihre feste, abweisende Ober-

31. Ebd., S. 137.

32. Ebd., S. 155-157

33. »A mesure que j'avance dans ce livre, je vois bien que c'est des choses qu'elle [0dile] a horreur. Elle y sent un danger mortel.« - »]e weiter ich in diesem Buch vorankomme, desto mehr sehe ich, dass es [0dile] vor den Dingen graut. Sie spürt dort eine tödliche Gefahr.« Ebd., S. 166.

34. »0dile marche à travers la campagne de France, et, petit à petit, avec précaution, elle délivre les choses.« - »0dile zieht über das Land, durch Frankreich, und, nach und nach, mit Vorsicht, befreit sie die Dinge.« Ebd., S. 219.

35. »Quand elle a heurté un meuble, elle le caresse de la main et, il n'y a pas encore longtemps qu'elle pleurait au bris d'un objet.« - »Wenn sie an ein Möbel stieß, streichelt sie es mit der Hand und es war noch nicht lange her, da weinte sie, wenn ein Gegenstand zerbrach.« Vgl. ebd., S. 175.

36. »[A] une certaine incidence de la lumière, par bonheur, les choses se 
fläche und damit ihre fremde, feindliche Wirkung. Die Metaphorik des Flüssigen, die den gesamten Text durchzieht, ist hier lesbar als ein Bild für die Auflösung der starren Grenze zwischen Subjekt und Objekt. Denn Odiles Bewusstsein erreicht nur »ce qui est soluble, ce qui, perdant sa densité, [...] se mélange à Odile aussi aisément que le sel à la salive «37 - »was löslich ist, was sich, seine Dichte verlierend, [...] genauso leicht mit Odile vermischt wie das Salz mit dem Speichel«. Wenn Margot Brink erklärt, es gebe in der Literatur der Moderne »Versuche, eine wenn auch alles andere als ungebrochene Gegenmagie zu diesem Erfahrungs- und Selbstverlust zu entwerfen " $^{38}$, dann lässt sich die Idee der >Befreiung ' der Dinge sicher als ein solcher verstehen. ${ }^{39} \mathrm{Da}$ neben steht jedoch die aktive Selbstversicherung in der Oralität. Über den Geschmackssinn und die Sprache, die in der >Höhle zwischen den Kiefern verortet sind, setzt sich bei Celli das schreibende Ich als Subjekt. Und dass dieses Subjekt kein fest umrissenes, selbstidentisches sein kann, ist im Bild der 'Zweiheit ‘ des Mundes, seinen beiden Kiefern, ebenfalls treffend gefasst.

Alexandra König

\section{Der heilige Magen: Sancho Panza}

Das Herz ist traditionellerweise der Ort, von dem vermutet wird, dass sich dort die menschlichen Gefühle befinden. Eine mittelalterliche Legende erzählt, wie ein Ritter, der sich in die Frau seines Nachbarn verliebte und, da er mit ihr schlief, gegen den ritterlichen Verhaltenskodex verstieß. Die Folge davon war, dass er von ihrem Mann getötet wurde, der des Herz des Liebhabers herausschneiden ließ und ein Mahl für die Frau zubereitete, die - ohne es zu wissen - das Herz ihres Geliebten

défont« - »Bei einem bestimmten Lichteinfall, lösen sich, zum Glück, die Dinge auf«. Vgl. ebd., S. 173.

37. Ebd., S. 79.

38. Vgl. Margot Brink: »Unzeitgemäßes im literarischen Feld der dreißiger Jahre: Nathalie Sarrautes Tropismes«, in: Helga Bories-Sawala (Hg.): Ansichten vom Frankreich der dreißiger Jahre, Bremen: Universität Bremen 2000, S. 100-111, hier S. 109.

39. Margot Brink zeigt auf, dass in Sarrautes Tropismes (1939) das Heil eines solches Gegenzaubers dagegen gerade verweigert wird. Dort sind die Dinge »so glatt und unzugänglich wie die Menschen« und stehen dem Protagonisten »fremd gegenüber«. Vgl. ebd., S. $108 f$. 